\title{
Respuestas al adiestramiento en industrias de alta tecnología mediante la educación abierta y a distancia
}

\author{
(Answers to training in industries of high technology through distance and \\ open learning)
}

\section{LILY STOJANOVIC}

\author{
Universidad Nacional Abierta \\ (Venezuela)
}

RESUMEN: Se presenta este trabajo como un planteamiento acerca de la pertinencia de la educación abierta ya distancia como nuevos enfoques de adiestramiento en industrias de alta tecnología. En el mundo tecnológico actual, éste requiere de respuestas de formación laboral ágiles y cónsonas con dicho ritmo. Con mayor frecuencia, las personas pasan de un trabajo manual a uno intelectual, lo cual implica ajustes sustanciales, a nivel de las calificaciones laborales que deben exhibir, así como de las organizaciones para que su sistema laboral se mantenga en condiciones funcionales. Ello implica un replanteamiento de los procesos de adiestramiento, para ajustarlos a las nuevas tendencias, y que permitan un impacto más efectivo en los resultados de los procesos productivos. En este sentido, este trabajo estudia la capacidad de respuesta que ofrecen la educac ón abierta y a distancia a la industria petrolera nacional, cuya fuerza laboral requiere de una reactualización constante, por su acelerado cambio tecnológico.

\section{Adiestramiento - educación a distancia en la industria - educación abierta en la industria.}

ABSTRACT: The increasing development of distance and open education in the last two decades rnade them to become an important part of most educational systems. Although initially concentrated at (he post secondary leve!, open and distance education projects now exist at the technical and vocational leveis, in (he form of job training, in the private as in the public sector. lncreasing!y, working people chan ge from manual to intellectual work which implies substantial changes in iheir labor califications, as in the working organiza non, specially in ihe last one, in order lo keep the working system in functional conditions. This makes necessay lo restate ihe training process, lo adjust it lo (he new iechnological tendencies of industries. The essay cha racterizes the possibilities that offer distance and open education as an answer for training, an llustrates it through the Venezuelan national oil industry. 


\section{training through distance education; training (hrough open education Educational answers to industrial training.}

Vivimos un período histórico caracterizado por un desarrollo vertiginoso de la ciencia y de sus aplicaciones tecnológicas. Al mismo tiempo, existe una insuficiente capacidad para gestionar socialmente estas aplicaciones. Sabemos de la importancia de las innovaciones, las transferencias y el desarrollo del sistema Ciencia-Tecnología, y sin embargo, en la medida que aquellas se van produciendo, y generando diversos problemas colectivos, la sociedad encuentra dificultades en cambiar con la rapidez suficiente para hacer frente a estos problemas y encontrar soluciones efectivas.

Las tendencias tecnológicas y sociales, actuales estan incidiendo fuertemente en los sistemas de producción industrial, y por consiguiente en la mayoría de sus empleos, los cuales están cambiando en su naturaleza muy rápidamente. Ello genera, entre otros, dos grandes problemas:

a) la capacitación que requieren estos empleos para poder dar respuestas válidas y oportunas en el mercado laboral; y

b) el mejoramiento de la calidad de vida laboral de todo trabajador, para hacerlo más eficiente con respecto a su trabajo, y lograr que sienta una mayor satisfacción por lo que hace.

Aún cuando se acepta la importancia que tiene el mundo del trabajo, este no posee, sin embargo, desde el punto de vista de la educación formal, un lugar propio, como objeto de reflexión técnico-pedagógica. Esto se explica, en parte, debido a la poca relación que siempre ha existido entre trabajo y educación, ya que se ha considerado que, criterios tales como economía y eficacia, no son muy aplicables al mundo educativo.

Coincidiendo con Sarramona (1990), es notoria la trascendencia que tiene la formación laboral para los sujetos y la comunidad en general. El trabajo, es un ámbito fundamental para la formación permanente, ya que constituye una actividad cotidiana, fuente de realización y frustraciones personales. Por otra parte, el nivel de formación que poseen los sujetos, determina la calidad de la producción.

Los cambios en el mundo del trabajo alteran necesariamente la propia naturaleza de la formación. En este sentido, el entrenamiento laboral se nos presenta como una necesidad apremiante en una economía sometida a un proceso de constante cambio, que se traduce a nivel industrial, en la incorporación de nuevos medios técnicos, nuevos métodos de producción y de gestión, que obligan a redefinir las tareas propias de cada puesto de trabajo.

En este sentido, no se puede seguir concibiendo a la formación como un simple instrumento adecuador de la mano de obra, ya que ella tiene un papel estratégico en relación a las transformaciones que se están dando en el mundo del trabajo. Por una parte, observamos que aumenta la productividad, con una tecnología cada vez más perfeccionada;se maximizan los beneficios y la rentabilidad por unidad de producto, lo cual exige nuevas respuestas de calificación profesional.Por otra parte, aumenta la necesidad de realizar tareas que mejoren la calidad de vida y que fomenten un bienestar lo más completo a quienes se desempeñan en el mundo laboral. 
Ambas exigencias no se pueden resolver únicamente a través de la educación presencial, tanto por razones de costo, como por limitaciones de tiempo y de espacio. El imperativo de adecuar la educación permanente del adiestramiento y capacitación profesional a las exigencias del cambio tecnológico, impone una nueva concepción de la educación en general, y en particular del adiestramiento, más dinámico y conexo con la realidad del mundo del trabajo. En este sentido,y de acuerdo al de aplicación y difusión mundial, la Educación a Distancia (ED) y Abierta, constituyen hoy día opciones relevantes y pertinentes para trascender los límites espaciales y temporales de la enseñanza presencial. Ambas opciones especialmente cuando se integran, pueden cubrir las exigencias planteadas, dada su naturaleza flexible para lograr la personalización del proceso didáctico y su potencialidad para proporcionar la generalización de la transferencia que ha de tener el adiestramiento.

\section{OCUPACIÓN Y PROFESIÓN}

Actualmente los procesos productivos se caracterizan por su fragmentación $\mathrm{y}$ descentralización, lo cual se refleja en trabajos parciales, subcontratos, modificación del mercado de trabajo, etc. Ello requiere cambiar el enfoque de la formación ocupacional tradicional, unidireccional, hacia un enfoque múltiple, ya que el empleo es, hoy por hoy, una variable más dentro del entorno socio-economico y productivo, que se reestructura permanentemente debido al cambio tecnológico y social que lo acompaña.

Por ello, la especialización tecnológica no es suficiente. Cuando comprobamos que muchos conocimientos se vuelven obsoletos y que un número de destrezas de los curijeula de las formaciones profesionales pierde vigencia, nos vemos en la obligación de integrar la técnica como una dimensión de la cultura básica.Una formación ocupacional exclusivamente profesionalizante se condena a sí misma a ser, segun Juver (1989) el aparcadero de los sin salida autónoma en mercado de trabajo.

Hoy en día, el concepto de profesión ha cambiado por uno más versátil que el de ocupación. Es preciso, que paralelo a las calificaciones específicas, el io trabajador adquiera también esquemas interpretativos, funcionales y rlque le permitan responder a situaciones profesionales diversas, así coanticiparse a eventualidades, tales como, el cambio continuo de trabajo a lo largo de si vida.

La formación se convierte no sólo en una oportunidad para que el individuo adquiera esa polivalencia necesaria y una mayor capacidad para organizar su propia formación, sino que se vuelve una protección relativa, ya que el trabajador requiere enfrentarse a nuevas realidades sociológicas, derivadas del cambio social y productivo. En este sentido, el aprendizaje debería orientarse hacia:

a) Una comprensión de los procesos

b) La adquisición de recursos capaces de reforzar su equilibrio psíquico y su propia identidad cultural; y

c) El uso de instrumentos que le permitan encontrar "sentido" a las complejidades de la vida cotidiana, tan llena de informaciones y nuevos conocimientos. 
No es suficiente con proporcionar experiencias laborales efímeras, ni adiestrar en especializaciones y destrezas, ya que el conocimiento y la continua modificación de saberes y técnicas adquieren mayor relevancia. Hay que formar por tanto en la iniciativa, espíritu crítico, autonomía y capacidad de razonar y pensar.Las demandas que requieren las nuevas concepciones de ocupación, definen cierto perfil, que podría sintetizarse como:

\section{A. Capacidad de interpretación y anticipación:}

Un programa de formación ocupacional debe ser capaz no sólo de plantear- se adquirir conocimientos y destrezas, sino el preparar al trabajador en la capacidad de observación y reacción frente a los imprevistos, y que sepa prevenirlos, a través de actividades de «anticipación». Cualquier programa innovador de formación ocupacional debe desarrollar actitudes que afectan a todos los conocimientos tecnológicos de base: los instrumentos de análisis sobre problemas y situaciones concretas requieren constante entrenamiento y dominio de operaciones mentales avanzadas. Si se fundamentara la formación sólo en destrezas o conocimientos que pueden ser substituídos por las nuevas máquinas y nuevas tecnología, no estaremos preparando para el futuro.

\section{B. habilidad para operar la abstracción:}

La incorporación de nuevas tecnologías en los procesos de producción hace disminuir el número de trabajadores necesarios en producción, y hace crecer el número de empleados relacionados con la gestión, tratamiento de la información, etc. Existe una cierta tendencia hacia el trabajo más abstracto, utilizando lenguaje e indicaciones, más que objetos materiales. Ello no quiere decir que los viejos procedimientos se eliminen, sino que se requiere cada vez más, un esfuerzo mental importante orientado hacia la comprensión de mensajes formales. La presencia de idiomas tecnológicos, la separación entre centros de decisiones, áre48 Respuestas al adiestramiento en industrias de alta tecnología mediante la educación abierta a distancia as de administración, gestión, con el lugar donde se utilizan los medios de producción, la comercialización, distribución, etc. implica un alejamiento psicológico y h'sico de los trabajadores respecto a su objeto de trabajo. La capacidad de abstracción se hace más significativa en la medida que las informaciones se presentan por diferentes medios de comunicación. Ello aumenta el papel de la formación permanente en la propia empresa para que los trabajadores aprendan la habilidad de utilizar correctamente mediaciones abstractas, que les permitan responder correctamente y tomar decisiones más acertadas.

\section{Capacidades y saberes que estimulan una mayor Calidad de Vida Laboral (CVL)}

Existe una serie de competencias, conocimientos y destrezas como son: capacidad de razonar para desarrollar funciones de relación y comunicación, relaciones interpersonales, capacidad de proyectar a través de la organización, conocimientos orientados hacia la solución de problemas de grupo, analfsis y toma de decisiones, 
creatividad,capacidad de lectura e interpretación; integrar conocimientos tradicionales con conocimientos modernos, uso correcto de lenguaje técnico; manejo del idioma propio, como idiomas extranjeros.

Las nuevas concepciones de ocupación constituyen condiciones nuevas y valores sociales que generan cada vez mayor influencia en los ámbitos de trabajo, lo cual ha originado el concepto de CVL. Esto a su vez debe reflejarse en los nuevos procesos de adiestramiento. Estas condiciones exigen competencias tales como:

a) Trabajo autónomo y con suficiente iniciativa

b) Organización y planificación del propio trabajo

c) Trabajo en equipo (procesos productivos de grupo)

d) Razonamiento lógico y de síntesis

e) Fácil desenvolvimiento en las relaciones interpersonales

Otras competencias:

Capacidad de gestión y participación en eventos nacionales e internacionales, capacidad para localizar información, interpretar datos, elaborar y emitir juicios. Por consiguiente, los problemas de adiestramiento que enfrentan actualmente las industrias de alta tecnología, no pueden ser resueltos por las formas tradicionales de entrenamiento técnico y teórico, fuera del ambiente de trabajo, que ha sido la característica más destacada de muchas escuelas técnicas industriales. Se requieren respuestas adecuadas a estas situaciones que generan las nuevas formas de producción y las demandas sociales, expresadas a través de los conceptos de CVL.

Cualquier estrategia nacional de desarrollo tecnológico que se quiera adoptar, tendría que tomar en cuenta dos aspectos básicos: capacitar al sector industrial para que cuente con los mecanismos y sistemas idóneos en la identificación y formulación adecuada de sus necesidades en materia de nuevo conocimiento; y el desarrollo de una capacidad de respuesta válida a las necesidades formuladas por el sector productivo, adecuada, no sólo en cuanto al comportamiento tecnológico de la misma, sino en cuanto a los factores de costo y tiempo de la respuesta.

\section{RESPUESTAS ACTUALES AL ADIESTRAMIENTO}

A fin de hacer frente a la creciente necesidad de actualizar las destrezas de los trabajadores en su sitio de trabajo, las corporaciones han aumentado fuertemente su educación y entrenamiento en los últimos años. En este sentido, puede afirmarse que la complejidad de la tecnología en el mundo moderno exige la transformación constante del mundo empresarial. Los requerimientos y niveles de exigencia de calificación de la fuerza de trabajo se transforman y aumentan constantemente.

Sin embargo, se observa que por lo regular, las corporaciones proveen a sus empleados tanto de educación remedial como entrenamientos técnicos muy sofisticados . La necesidad de entrenamiento en el lugar, y en relación con el trabajo, se ha incrementado a tal punto que, las corporaciones, hoy día, cada vez más, planifican sus propios programas de formación. Esto, en gran medida, ha forzado a las instituciones educativas 
a buscar un mayor intercambio de sus relaciones con las empresas, para el desarrollo y administración de programas de entrenamiento y reentrenamiento.

El entrenamiento en el sitio de trabajo se ha incrementado fuertemente en los últimos años, a tal punto, que en ciertos países como USA, el gasto para ello, es casi igual que el de la educación pública.

No obstante que las actividades de entrenamiento en las industrias han sido bastante amplias, su alcance no ha sido generalizado a todos los empleados. Las oportunidades de adiestramiento, están distribuidas en forma dispareja, tanto en las industrias y ocupaciones, como en los grupos de empleados. En general, las industrías con empleados que poseen las mejores destrezas, o industrias altamente especializadas (bancos, seguros,...) tienden a proveer mayor entrenamiento, que las empresas más pequeñas. Por lo regular los beneficiarios son empleados de cuello blanco, entre 25 a 40 años, y profesionales. Los empleados de cuello azul (obreros) reciben, en cambio, más bien instrucción informal y en el trabajo (Gordon, 1987).

Algunas empresas limitan sus entrenamiento a la adquisición de destrezas técnicas, mientras que otras son más amplias, e incorporan también el desarrollo personal.

En la medida en que las compañías planifican estrategias de reentrenamiento para sus empleados, muchas requieren de educación remedial, antes de proceder a cualquier forma de instrucción. Estas compañías se han dado cuenta de que los trabajadores con bajos niveles de destrezas son deficientes en su productividad, por lo que sus productos son de baja calidad, y sufren por lo general mayor cantidad de accidentes, lo cual justifica la instrucción remedial (Lee, 1986).

No obstante la mayoría de las compañías prefieren conducir ellas mismas sus programas de entrenamiento, dejando el problema de reentrenamiento por cuenta propia de los empleados, quienes recurren a instituciones educativas externas. Existen algunos programas de colaboración, que varían tanto en su alcance como en su foco de atención. En algunos casos, los proyectos son en gran escala y a largo plazo, mientras que otros, se basan en necesidades a corto plazo. Algunos proyectos incorporan personal que ha sido desplazado por la automatización, o porque han cambiado las condiciones de la organización; otros, ayudan a los empleados a mejorar sus destrezas actuales.

Aunque el entrenamiento y desarrollo de los empleados no han sido motivo de gran preocupación en el pasado, sí lo son ahora que existen las presiones de una competencia mayor, así como el gran desarrollo de la tecnología, lo cual ha determinado, cada vez más el que se tomen en cuenta las facilidades de entrenamiento en los contratos colectivos de trabajo.

Recientemente, la naturaleza de las relaciones entre empresa e instituciones educativas se ha profundizado. En países altamente industrializados, (USA, Inglaterra, Alemania,etc) las instituciones educativas ven a las empresas como clientes y les proporcionan entrenamiento, sobre una base contractual. Por ejemplo, la Hewlet Packard, contrata con la Universidad de Stanford USA, y otras, como el Massachussets Institute of Technology. Otras corporaciones contratan instituciones educativas en una escala mas pequeña. Por ejemplo la IBM y su programa de reentrenamiento en un Community College. 
Sin embargo, a pesar de los múltiples ejemplos que ilustran la relación entre educación y empresa, en países desarrollados como USA, se considera que el principal problema aún, es la falta de una buena relación entre la institución educativa y la industria (Zemke, 1985). Por otra parte, las instituciones de educación post secundaria, por lo general no están preparadas lo suficientemente para desempeñar un rol importante en el entrenamiento que requieren las industrias (Fields, 1986). Consideran así mismo, que las instituciones educativas, no son lo suficientemente flexibles ni prácticas para diseñar programas de reentrenamienme encajen oro2resivamente con las necesidades de las empresas e industrias.

Evidentemente que el incremento y sabio uso de la tecnología tanto para la ción como para el entrenamiento, es fundamental para el desarrollo. En el $78 \%$ de todos los empleos en USA fue en industrias de servicio y la tendencia parece acrecentarse a futuro (The Economist, 1994). El trabajo, igualmente seguirá cambiando; los que se gradúan actualmente en el tercer nivel, deberán reentrenarse por lo menos cinco veces en toda su vida laboral.

Nuestro contexto nacional, no escapa a la realidad de atender la creciente necesidad de reentrenamiento de las profesiones, lo cual se hace particularmente evidente, por su significado en el mercado nacional e internacional, en la industria petrolera, ya que ésta representa un sector de alta tecnología.PDVSA (Petróleos de Venezuela) constituye una de las más grandes corporaciones productivas de petróleo, dentro del contexto de la globalización, caracterizada por una acelerada dinámica de cambio, y mercados, cada vez más competitivos. Inicialmente, en 1976, se creó el INAPET (Instituto de Adiestramiento Petrolero y Petroquímico), el cual realizó su labor a nivel de obreros calificados y supervisores de primera línea. Su programa permitió preparar operarios en los principales oficios requeridos en la industria. En 1983, se creó el CEPET (Centro de Formación y Adiestramiento Petrolero y Petroquímico), el cual integró actividades del INAPET y FONINVES (Fondo destinado a la formación de personal en el área de hidrocarburos). Posteriormente, se creó el CIED en 1995, para servir como la Universidad Corporativa de PDVSA.

Igualmente, la IPPCN (Industria Petrolera Petroquímica Carbonífera Nacional) ha realizado convenios de asistencia tecnológica, y de intercambio de personal con calificadas empresas del exterior, y ha fomentado acuerdos con instituciones educativas, tanto nacionales como internacionales, y a través de las formas instruccionales de talleres, seminarios, videoconferencias, simposios, charlas, etc.

\section{TENDENCIAS E INNOVACIONES}

Como ya se indicara con anterioridad, a las dinámicas condiciones y necesidades de la industria moderna de alta tecnología, se requieren nuevas respuestas de adiestramiento. En este sentido,algunas tendencias a nivel mundial, señalan la con. veniencia de una más estrecha relación trabajo- aprendizaje, lo cual significa que una parte importante del adiestramiento debe realizarse en el sitio del trabajo.

En el caso de la industria petrolera venezolana, el adiestramiento, en la década de los 90 se ha caracterizado por: 
a) Altas inversiones en mejoramiento de personal

b) Ënasis en la preparación para «aprender»y enseñar ; y

c) Conversión de talleres en aulas.

Dentro del amplio panorama de posibilidades para el entrenamiento, se destacan actualmente, a nivel mundial, la educación a distancia y la educación abierta. Ambas, pero especialmente la primera, se han extendido rápidamente debido a su adecuación a las necesidades de la industria moderna. Por ello se han convertido hoy día en los instrumentos más ágiles para dar respuestas al adiestramiento.Veamos ¿por qué?

Las ventajas de la aplicación de esta modalidad, se derivan de su gran flexibilidad, para que:

a) los estudiantes aprendan en situaciones de trabajo, sin grandes desplazamientos y costos, que resultan indispensables en algunas modalidades presenciales b) se desarrollen y mantengan sentimientos de pertenencia corporativa, al estimular una constante vinculación con el clima de trabajo de la empresa.

Esta flexibilidad permite alcanzar, cierta individualización del proceso didáctico, y facilitar el logro las transferencias de aprendizaje, cuando en el proceso se procede a la observación de casos, luego la generalización, para ir a situaciones aplicativas reales o supuestas, con lo que se materializa el «adiestramiento in situ».

La modalidad a distancia aplicada al adiestramiento moderno, requiere, por lo regular, de una combinación equilibrada con la modalidad «in situ» y con la flexibilidad propia de un sistema «abierto», y con un fuerte apoyo de avanzadas tecnologías educativas, ya que es poco frecuente un uso puro y exclusivo de la modalidad a distancia.

La incorporación «in situ», facilita aspectos tales como, aplicación práctica de teorías y conceptos, el manejo de equipos y procesos, destrezas motoras y relaciones interpersonales. Por otra parte, lo abierto introduce la moderna tendencia de que conjuntamente con el aprendizaje de conocimientos, destrezas y habilidades necesarias para una determinada tarea,es preciso alguna previsión acerca de las condiciones y aspiraciones del que aprende. Con ello, se podría dar cierto grado de individualización y de motivación, especialmente ésta última, ya que constituye factor esencial para lograr un aprendizaje eficiente y eficaz.

Existen dos elementos que son muy importantes en la ED. estas son: La autoinstrución y las interacciones personales.

Autoinstrucción: Se refiere al proceso en el cual, la persona que aprende no requiere de la presencia permanente de un instructor que le enseña directamente.

Interacciones personales: Diferentes a los contactos permanentes que se establecen usualmente en las clases magistrales. En la modalidad a distancia, la interacción personal puede ser necesaria, pero adopta formas muy diferentes. En primer lugar, suele ser esporádica y muchas veces, voluntaria, dependiendo de los grados de dificultad que encuentra cada estudiante. En segundo lugar, la función de estos contactos es la de tutoría y apoyo, y no la de proporcionar clases. En tercer lugar, dichos apoyos adoptan 
múltiples formas, y utilizan recursos muy diversos; puede tratarse de tutorías sobre conocimientos, o de orientaciones sobre formas de estudio o problemas personales. En esta multiplicidad se diferencia significativamente de las formas de relación que utilizaba la educación por correspondencia, que se centraba exclusivamente en la relación del estudiante con el material impreso.En la ED. y A. el participante puede interacturar con:

a) Un agente educativo

b) El grupo de colegas (participantes)

c) Los medios autoinstruccionales (textos, programas de computación, audiovisuales)

En lo que se refiere al «agente educativo», la aplicación de la ED requiere formar adecuadamente al supervisor de los procesos industriales, para que actúe como facilitador del aprendizaje. Esto requiere como paso previo la estructuración de programas de formación de facilitadores-supervisores.

En cuanto a su implementación, permite abaratar costos de formación a largo plazo, aunque requiere de inversiones iniciales significativas. Además evita los desplazamientos de los lugares de trabajo, lo cual genera ventajas de costo, y de pertenencia. La enseñana a distancia, no permite una actitud meramente de «presencia» ante el proceso formativo, sino que obliga a comprender y actuar en el mismo ambito laboral sobre el que se contextualiza la formación, Sarramona(1990).

\section{POTENCIALIDAD DE LA MODALIDAD A DISTANCIA:}

La educación a distancia ha evolucionado a través de lo que han denominado (Nipper, 1989; Kaufman, 1989; Bates 1995), las tres generaciones de la ED.La primera generación,con predominio del uso de un sólo medio tecnológico, (me. dio impreso), y total ausencia de una interacción entre los estudiantes, como lo fue la educación por correspondencia, de finales del siglo pasado. La segunda ge. neración, se caracterizó por un enfoque multimedia, con materiales diseñados deliberadamente para el estudio a distancia, pero en la cual, la comunicación aún estaban mediada por el tutor (no quien producía el material instruccional). Este fue el modelo que desarrollaron la mayoría de las universidades a distancia que surgieron en todo el mundo para esa época (predominante aún en el medio universitario venezolano, con los EUS-UCV;Estudios Universitarios Supervisados;y la UNA(Universidad Nacional Abierta).La tercera generación, es la que se basa en la comunicación de dos vías, a través de medios que permiten la interacción di. recta entre el estudiante a distancia y quien origina el material instruccional. A menudo se da entre estudiantes a distancia en grupos. La tecnología propia de esta generación, ha permitido una distribución más equitativa entre estudiante y docente y entre los estudiantes mismos. Esta progresión evolutiva ha permitido lo que señala Kaufman (1989) un mayor control por parte del estudiantes, oportunidades para el diálogo, énfasis en destrezas de pensamiento más que en comprensión, lo cual ha conducido a nuevos tipos de organizaciones educacionales. Pero esta última generación ha permitido desarrollar a partir de las nuevas tecnologías de información y comunicación lo que ha denominado Chacón, 1997, paradigma "Informático-telemático". Este paradigma que incluye algunos elementos exitosos del 
enfoque modular multimedia tales como presentación de información a través de diferentes medios, uso de tutores, evaluación automatizada, introduce nuevos elementos que convierten a la educación a distancia actual como la modalidad educativa integradora de los últimos avances tecnológicos al servicio educativo. El punto clave que diferencia este nuevo paradigma es:

..." el uso de las comunicaciones mediante la computadora (CMC) es el corazón del sistema de enseñanza a distançia, creando así un vehículo permanente para la comunicación y para disponer de grandes masas de información útil para los propósitos educativos También es una constante el uso de las teleconferencias de audio y o video, las cuales pueden estar mediadas por computadoras o no; sin embargo la evolución de esta tecnología indica que en el futuro la mayor parte de las teleconferencias de audio y video serán a través de computadoras, debido a que la digitalización y compresión de mensajes significa una ganancia neta en cuanto al costo..." (Chacón,1997, p. 101)

A pesar de la exagerada estimación que se ha hecho de los beneficios del uso de la computación en educación, se deben considerar tres desarrollos significativos como ha sido el de los multi media y la consiguiente aplicación educativa de las computadoras; el uso de las redes de computación con propósitos comunicacionales; y un cambio en la filosofía acerca del uso de las computadoras como máquinas de enseñanza, a herramientas de refuerzo para docentes y estudiantes.

Aunque las computadoras deben su nombre a la gran capacidad de procesar enormes y complejas masas de cálculos, hoy día su espectro de funciones es muy amplio, particularmente en las llamadas microcomputadoras, cuyo potencial respecto a las primeras es cada vez mayor. Entre éstas, pueden señalarse:

- gran capacidad de almacenamiento, a través del microproceamiento obtenido por la tecnología del "chip". Esto ha traído como consecuencia digitalizar audio, video y gráficos.

- desarrollo de multimedia: integración de video, audio y texto dentro de la arquitectura del computador - desarrollo de software, el cual permite el uso fácil de herramientas de computación, sin requerimiento de mucho entrenamiento profesional en computación para poder crear materiales de instrucción - aunque todavía altos, cada vez más los precios para adquirir un computador son más accesibles

- mayor capacidad en el establecimiento de redes a través de uso de servidores

- mayor versatilidad en el uso intercambiable de materiales en diversos tipos de computadores

- mayor facilidad en las "interfases", esto es, entre el usuario y la computadora, a través de comandos de voces, reconocimiento de voces, posiciones de manos para controlar la programación, y el desarrollo de la inteligencia artificial para la interpretación de la computadora a los comandos y requerimeintos humanos.

En este sentido la aplicabilidad de las computadoras en ED y aprendizaje abierto ha sido en las siguientes areas: 
- administrativas

- evaluación de estudiantes

- comunicaciones

- publicaciones electrónicas

- aprendizaje basado en computador

- distribución electrónica de materiales

Desde el punto de vista de la enseñanza existen dos vías de enseñar a través del computador: aprendizaje basado en el la programación del computador (ejemplo el multimedia) y la comunicación mediada a través del computador. En el primer enfoque, el que aprende a través de materiales prediseñados, interactúa a través de sus respuestas, incorporadas en los materiales y escogiendo rutas u opciones, a tarvés del material. El computador es capaz de utilizar las respuestas que da el estudiante para efectos de control y de retroalimentación. Así mismo, los programas de aprendizaje basado en el computador pueden evaluar al estudiante y servir de registro de su progreso, a los cuales puede recurrir el tutor o instructor.

Los avances tecnológicos más recientes, permiten incorporar, vídeo, audio, gráficos animados en forma integrada, lo cual ha determinado el desarrollo del multimedia (añadir el video disco, o el CD-ROM, al computador, amplía el rango de posibles aplicaciones educativas del mismo).

Si bien el uso de este enfoque se ha dado en la Open University de Holanda, la Open University de Inglaterra; la Fern Univesitat de Alemanía; en Canadá, su uso más generalizado ha sido en el mercado de entrenamiento. Van der Brande (1993) señala que en Europa la principal concentración de este enfoque se encuentra en el área de las finanzas y los seguros, la manufactura y los servicios públicos.

En el segundo enfoque, el que aprende tiene un mayor contacto con el que enseña y con otros pares; y a pesar de su distancia puede accesar la base de datos a través de las redes de información, extraer la información y almacenarla en su propio computador., para uso posterior. Igualmente puede conectarse con otros pares, o instructortes. En este sentido la interacción no es tanto con el computador, sino a través de él con otras personas, o con otras fuentes de información.

Si bien existe el debate entre si esta tecnología resultará en un nuevo paradigma educativo, o que sólo permite usar más efectivamente viejos paradigmas, es bien cierta las inmensas innovaciones que se han hecho con su aplicación, así como el constituir una de las tecnologías de mayor crecimiento, en término de su uso, por parte de docentes y estudiantes (Bates, 1995).

Ha habido una creciente expansión del uso de la comunicación mediada por el computador (CMC) en educación abierta y a distancia, desde los últimos años de la década de los ochenta. Wells (1982) ha descrito 93 instituciones a nivel mundial que han ofrecido cursos en veinte países diferentes, a través de este enfoque. Si bien se utiliza el correo electrónico para la comunicación entre estudiantes y tutores, y para la revisión de trabajos, la conferencia vía computador es la forma más generalizada de este enfoque. 
Este enfoque puede ser utilizado en una gran variedad de formas como adicional de un curso basado principalmente por material impreso, o instrucción cara a cara; puede también ser el medio principal y su uso puede igualmente ser opcional o el requerido por el curso.

Los beneficios instruccionales de la conferencia electrónica pueden resumir- en los siguientes:

- Desarrolla destrezas analíticas, de construcción y defensa de argumentos, permite organizar evidencia para apoyar un argumento, crítica de otros trabajos, etc.

- Refuerza el trabajo colaborativo, con énfasis en la interacción grupal, compartir conocimientos y experiencias, compartir un tópico para organizar discusiones en grupo, provee la oportunidad para la reflexión.

- Facilita el trabajo en proyectos de grupo, que pueden ser igualmente evaluados, de acuerdo a la contribución de cada uno de los participantes.

- Permite la generación de conocimientos creativos, vinculación y estructuración (Harasim, 1990).

- Maximiza el conocimiento y las experiencias de los participantes, lo cual es muy importante para audiencia de estudiantes adultos, quienes poseen conocimiento y experticia que muchas veces no posee el experto en una asignatura.

- Vincula las experiencias de los diversos participantes, permitiendo compartir diferentes pespectivas desdes diversas culturas

- Desarrolla destrezas de redacción, particularmente como herammienta comunicacional entre estudiantes dispersos

- La conferencia provee una ayuda inmediata, sea de tutores o de pares en tópicos específicos, a veces puede ser un estudiante quien provea una mejor respuesta que el tutor

Este paradigma informático-telemático comprende diferentes modelos de aprendizaje a distancia, que han sido reseñados por Chacón, 1997 como:

a) Sistema modular enriquecido; (se sigue utilizando material impreso, asesorías individuales o en grupo y recursos audioviduales, pero se incluyen el correo de voz y correo electrónico.

b) Sistema basado en teleconferencias (conferencias dictadas por uno o más expositores expertos que se transmiten en diversos puntos con el uso de redes satelitales). c) Sistema multimedia individual (Enseñanza asistida por computador, video interactivo, o adiestramiento basado en el computador). En todos ellos, se ha potenciado el computador como auxiliar de la enseñanza, amplíandose su uso con el CD-ROM. d) Sistema de enseñanza en línea. Consiste en la creación de comunidades o grupos virtuales con fines de aprendizaje a través de redes en ambientes intranet o internet.

Dentro de este modelo, los docentes preparan la información a través de distintos medios de presentación digital que permiten utilizar texto, datos, imagen, sonido, y video. Los estudiantes interactúan con los docentes y entre ellos a través de las redes, y 
así mismo presentan las evaluaciones. Se utilizan también ambientes virtuales que simulan los sitios usuales de enseñanza. Para ello se utiliza el sistema de aprendizaje distribuido, constituido por un pa. quete de software capaz de coordinar múltiples cursos, estudiantes y docentes. Los recuros para presentar la información son boletines, listas de usuarios, áreas de conversación, foros , libros electrónicos, páginas Web, cuestionarios de evaluación.

Un ejemplo concreto de este último es la industria petrolera nacional, la cual ha reconocido su creciente importancia, incluyendo la ED en sus políticas para adiestramiento. En tal sentido el CIED ha señalado, que en los próximos años, su acción se orientará hacia el incremento progresivo de programas de adiestramiento a distancia, la incorporación de nuevas tecnologías para la formación artesanal especializada, y mayor difusión del adiestramiento, asistido por la computación y otras ayudas no convencionales. Para alcanzar su objetivo de "investigación de las tecnologías pedagógicas más recientes y diseño de material educativo, utiliza los más avanzados recursos en comunicaciones, como videoconferencia en tiempo real, nacional e internacionales , así como la presentación y acceso a cursos, y material didáctico a través del internet corporativo" (CIED, Universidad Corporativa, 1997).

Para determinar la pertinencia del uso del enfoque educativo a seguir en las industrias de alta tecnología, habría que analizar cuales son las situaciones de enseñanza individuales y grupales,propias de estas industrias. Podría infererirse de los estudios al respecto (Chacón; 1997, Paulsen, 1995) que los ejemplos de enseñanza más pertinentes se orientan en los enfoques c) y d)

La industria petrolera venezolana, enfrenta el desafío que significa para el próximo milenio renovar y actualizar su fuerza de trabajo. Actualmente la empresa ha emprendido un proyecto de sistema de aprendizaje virtual, aplicado a la División de Exploración y Producción. El propósito de este sistema es consolidar las competencias de distintos profesionales de la ingeniería, geólogos, geofisicos, a través de un aprendizaje acelerado, a la vez que constituir una herramienta de conocimiento para la información técnica y prácticas de operación en los campos petroleros.

El sistema de aprendizaje virtual ilustra la aplicación de la situación de enseñanza en línea, del paradigma informático-telemático, y que aplicado a este caso consiste en tres estrategias básicas: instrucción individual en línea, aprendizaje colaborativo con tutores y pares; y conocimeinto organizacional. El ciclo de aprendizaje de cada participante implica el diagnóstico de competencias previas, contrato de aprendizaje, interacción en línea con conocimiento práctica y básico, análisis de fuentes de datos adicionales, evaluación a través de tareas simuladas y record de resultados. Durante todo este ciclo, el participante tiene acceso a un tutor individual y conocimiento de apoyo, por parte de expertos. (Chacón, y Brand, 1999).

\section{CONCLUSIONES:}

A) En relación a las tendencias de desarrollo de las industrias de alta tecnología: Estas están modificando significativamente sus estructuras organizativas, tecnologías aplicadas a la producción, formas de trabajo y aspectos de clima organizacional. Sus 
componentes eléctricos, tienden a ser sustituidos por una creciente automatización que altera significativamente las competencias requeridas en los principales empleos. Por otra parte, la participación, actitudes y valores de los empleados son determinantes en la calidad de vida laboral, así como en su rendimiento.

B) En relación a las necesidades de adiestramiento: Los continuos cambios que se producen en dichas industrias obligan a modificar los sistemas tradicionales de adiestramiento, ya que se requiere:

- un adiestramiento permanente acorde con las solicitudes de producción;

- centrado principalmente en el sitio de trabajo;

- incorporar las «tecnologías informativas» que más se ajusten a las situaciones instruccionales correspondientes;

- tomar en cuenta condiciones que favorezcan una «calidad de vida laboral», vinculada a la calidad total.

C) En relación a algunas respuestas para el adiestramiento: Deben redefinirse los procesos de detección de necesidades, a fin de que reflejen los verdaderos requerimientos de la industria. Los programas de entrenamiento deben enfatizar:

- procesos industriales y de aprendizaje; y

- condiciones y aspiraciones de los participantes. En este sentido, la ED facilita:

- la posibilidad de que el adiestramiento se realice en el sitio de trabajo

- recurrir a los especialistas más destacados en la industria

- integrar teoría y praxis, mediante el uso de estrategias instruccionales apropiadas

- incorporar modernas tecnologías informativas aplicadas al adiestramiento

- la unificación corporativa de programas de adiestramiento

- adiestrar masivamente a determinado personal esparcido en diversos puntos geográficos

- proporcionar materiales instruccionales a los participantes

- disminuir significativamente costos de adiestramiento.

La educación abierta, es principalmente un objetivo o política educativa (Bates, 1995), que busca proveer el aprendizaje de manera flexible y adaptado a las condiciones y limitaciones de los que aprenden.Permite combinar diversas modalidades. Puede incluir ED o depender de otras formas flexibles de aprendizaje, como sería estudio independiente combinado con enseñanza presencial. Su princi. pal ventaja se refiere a facilitar que las estrategias y procesos de la industria se integren con los intereses 
individuales del participante. Ha sido aplicada, principalmente en programas con una función instrumental, pero igualmente pemite aplicarse a programas con funciones cognitivas y expresivas. Y aunque difiere de la educacióna distancia en que no es propiamente una modalidad educativa, comparte con la ED el constituir un medio alternativo para proveer entrenamiento en forma distinta a la convencional.

Educación a distancia y educación abierta generalmente se verán combinadas, siendo una cuestión de grado su nivel de aplicación al contexto educacional de que se trate.

Debido a que el conocimiento hoy día se expande tan rápidamente, ello afectará igualmente el rol de los docentes, el cual consistirá predominantemente en ser consejeros, administradores y facilitadores del aprendizaje, más que proveedores de la información, a la cual podrá accederse a través de las telecomunicaciones. El docente, deberá ser diestro en "navegar" en las fuentes de información, y poseer destrezas en el procesamiento y análisis de la información. En el caso de la industria, serán los expertos profesionales quienes vayan a asumir un papel docente significativo, sin haber sido profesionales de la docencia. Quien posea experticia en el conocimiento será posiblemente quien realice funciones educativas y de entrenamiento. Por ello el aprendizaje a futuro requerirá de una combinación de diversos modelos educativos, en los cuales la ED y abierta seguirá jugando un papel cada vez más importante, ya que el aprendizaje seguirá siendo un proceso individual y la comunicación de dos vías se extenderá a otros pares y docentes, Ello puede constituir la base para crear nuevos modelos curriculares que optimicen al máximo las potencialidades de las nuevas tecnologías.

La incorporación de nuevos medios, como los arriba descritos, en el enfoque de educación abierta y a distancia, sustituye el ciclo rígido de un sólo sentido, del enfoque tradicional, según el cual el mensaje instruccional se elabora de antemano, se produce mediante diversos medios y se administra a los participantes de acuerdo a los criterios establecidos en la fase de planificación, por ciclos múltiples de planeamiento y selección de materiales, producción, entrega, asesoría y evaluación, los cuales se deciden progresivamente según ocurre el proceso mstruccional, (Chacón, 1997), con lo cual la educación a distancia adquiere un sentido más dinámico, ajustado a las características de las necesidades de entrenamiento actual, y en las cuales las técnicas de enseñanza en línea reproducen en un nuevo concepto las técnicas de enseñanza grupal, tal como se desprende del estudio que ha realizado Paulsen (1995) sobre las distintas técnicas de enseñanza utilizadas en los ambientes de redes.

\section{REFERENCIAS BIBLIOGRÁFICAS}

BATES, A. (1995). Technology, Open Learning and Distance Education. Routledge. London.

CASAS, A. M. (1991) .Conferencia:Tendencias e innovaciones en el Adisestramiento Industrial "Jornadas de Adiestramiento CEPET. Zulia.

CENTRO INTERNACIONAL DE EDUCACIÓN Y DESARROLLO (CIED). (1997). Prospecto informativo 
CHACÓN, F. (1997). Asuntos. Un nuevo Paradigma para la Educación Corporativa a Distancia. 1 Centro Internacional de Educación y Desarrollo (CIED). Año 1 (2), 199119

CHACÓN, F.; R. BRANDT. (1999).Developing the work-force of the Petroleum In4ustry through Virtual Learning. Poster presentado en la Décimo Novena Conferencia mundial sobre Educación A Distancia. ( ICD). Viena.

ECONOMIST, TIIE (1994). Schools Brief. The Manufacturing Myth Vol. 330. (March, 19-20).

GOLDSTEIN, 1. (1986). Training in Organizations: Needs Assesment, Development and Evaluation. Monterrey, Cal. Brooks Cole.

GORDON, 3. (1987). Where the training Goes. Training: The Magazine of Human Resources Development.(October).

HARASIM, L. (1990) (Ed.) On-Line Education: Perspectives on a New Environment, New York. Praeger.

JACOBS, R. L.; T.D. McGIFFIN. (1987). A Human Performance System Using a Structured On the Job-Training Approach. Performance and Instruction. 25 (7) 9-11.

JUVER, D. (1989). La Formación Ocupacional. Edit. Popular. Ministerio de Educación y Ciencias. Madrid.

KAUFMAN, D. (1989) En SWEET, R. (Ed.) Post Secondary Distance Education in Canada.: Policies and Priorities, Athabasca: Athabasca University. Canadian sutdies for distance education

LEE, L. (1986) Literacy Training: Hidden Need Training. The Magazine of Human Resources Development. (September).

NIPPER, S. (1989). En A. BATES. Technology, Open and Distance Laarning. Routledge.London. PAULSEN, M.(1 995). The Online Repon on Pedagogical Techniques for Computer-Mediated Cernmunication. College at http:www.nki.no.Norway.

SARRAMONA, J. (1990). La Educación a Distancia en la Formación Laboral. En A. VILLARROEL; F. PEREIRA (Eds.) La Educación a Distancia Desarrollo y Apertura. International Council for Distance Education.

STOLOVITCH H.; E. KEEPS (Eds.) (1992). Handbook of Human Perforniance Technology. National Society for Performance and Instruction. Jossey-Bass.

ZEMKE, R. (1985) Industry Education Cooperation. Oid Phrase with Strange New Meaning, Training: The Magazine of Human Resource Development. (July). 


\section{PERFIL ACADÉMICO DEL AUTOR:}

Lily Stojanovic. Maestría en Educación (Stanford USA). Profesora Titular de la Universidad Central de Venezuela (UCV) y de la Universidad Nacional Abierta (UNA) como diseñadora de instrucción. Posee experiencia en estudios a distancia, desde el inicio de las Estudios Universitarios Supervisados (EUS) en la UCV. Ha publicado dos libros sobre evaluación de aprendizajes y elaboración de materiales instruccionales; y numerosos artículos sobre diseños instruccional, evaluación y educación a distancia.

El más reciente, en colaboración con Miguel Casas A. De Vancouver 82 a Viena 99 RIED 1999:2.

Teléfono: (58-2) 7814051

Fax: (58-2) 7936802

E-mail: lilystojanovic@yahoo.com This email address is being protected from spam bots, you need Javascript enabled to view it 